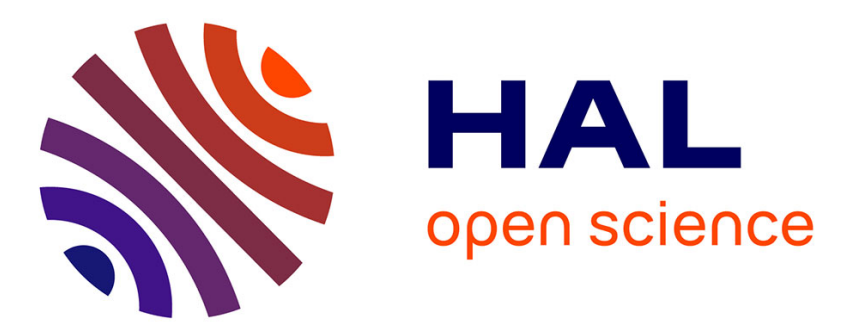

\title{
Identification of brain regions involved in epilepsy using common spatial pattern
}

Samareh Samadi, Ladan Amini, Hamid Soltanian-Zadeh, Christian Jutten

\section{To cite this version:}

Samareh Samadi, Ladan Amini, Hamid Soltanian-Zadeh, Christian Jutten. Identification of brain regions involved in epilepsy using common spatial pattern. SSP 2011 - 2011 IEEE Workshop on Statistical Signal Processing, Jun 2011, Nice, France. pp.829-832. hal-00605699

\section{HAL Id: hal-00605699 \\ https://hal.science/hal-00605699}

Submitted on 4 Jul 2011

HAL is a multi-disciplinary open access archive for the deposit and dissemination of scientific research documents, whether they are published or not. The documents may come from teaching and research institutions in France or abroad, or from public or private research centers.
L'archive ouverte pluridisciplinaire HAL, est destinée au dépôt et à la diffusion de documents scientifiques de niveau recherche, publiés ou non, émanant des établissements d'enseignement et de recherche français ou étrangers, des laboratoires publics ou privés. 


\title{
IDENTIFICATION OF BRAIN REGIONS INVOLVED IN EPILEPSY USING COMMON SPATIAL PATTERN
}

\author{
S. SAMADI $I^{1,2}$, L. AMINI $^{1,2}$, H. SOLTANIAN-ZADEH ${ }^{2,3}$ and C. JUTTEN ${ }^{1}$ \\ 1- GIPSA-LAB, Grenoble INP, Domaine universitaire- BP 46, F-38402 Grenoble Cedex, France. \\ 2- Control and Intelligent Processing Center of Excellence (CIPCE), University of Tehran, Tehran, Iran. \\ 3- Radiology Image Analysis Laboratory, Henry Ford Health System, Detroit, MI 48202, USA.
}

\begin{abstract}
In this paper, we aim to identify the regions involved in epilepsy from intracerebral EEG (iEEG) of patients suffering from focal epilepsy. Identification of regions involved in epilepsy is important for presurgery evaluations. The proposed method is based on common spatial pattern (CSP) using two types of time intervals: 1) periods including interictal epileptiform discharges (IED), and 2) periods excluding IEDs or abnormal physiological signals. The method is applied on the iEEG recordings of one seizure-free patient after resective surgery. The results are compared with seizure onset zones visually inspected by the epileptologist. The congruent IED regions with visually detected seizure onset zones are encouraging results. Moreover, the application of CSP method for the identification of IED regions seems interesting as this method is fast and simple.
\end{abstract}

Index Terms - Common Spatial Pattern, General Eigen Value Decomposition, Epilepsy, Intracerebral EEG

\section{INTRODUCTION}

Drug-resistant epileptic patients are recommended to undergo resective surgery. The aim of this surgery is to remove the brain regions responsible for the seizures without creating new neurological deficits [1]. Different dysfunctional regions are studied for presurgery evaluations. Two important dysfunctional regions are: 1) seizure onset zone (SOZ), and 2) interictal epileptiform discharge (IED) region. Localization of SOZ is difficult since seizures are rare events and may not provide robust results. As such, prediction of SOZs from IED regions is valuable. However the relationship between SOZs and IED regions is still an open issue.

In this paper, our goal is to identify IED regions from intracerebral EEG (iEEG) recordings. We aim to identify the

C. Jutten is also with Institut Universitaire de France,103, bd SaintMichel 75005 Paris, France.

The authors gratefully acknowledge P. Kahane, L. Vercueil, O. David, and L. Minotti (from Grenoble Institute of Neuroscience, U836 INSERM, and/or Neurology Department, CHUG, Grenoble, France) for providing the data, interpretations, and their helpful assistance in this study. temporal sources, and eventually spatial patterns which are related to the differences between two brain states: 1) IED state, and 2) non-IED state. Thus, two corresponding periods of iEEG recordings are studied: IED and non-IED time intervals. IED time intervals are the periods including IED signals, while non-IED time intervals are the periods excluding any IED or abnormal physiological signals.

To extract the discriminative sources between IED and non-IED states, common spatial pattern (CSP) method seems to be an appropriate choice. CSP is a classification method that was first proposed by Fukunaga and Koontz [2]. The objective of this method is to extract patterns which represent the difference between one class of data and another. CSP is formulated as an optimization problem in which maximizes the ratio of variance between two classes [3]. This method has been widely used in many contexts especially in Brain Computer Interface (BCI) [4].

Here, we estimate the IED regions using CSP. CSP is known to be sensitive to the outliers [4]. However in our application as the number of iEEG samples is large enough this drawback is not problematic. The method is applied on one iEEG recording of a seizure-free patient after resective surgery. The estimated IED regions are compared with visually inspected SOZs by the epileptologist.

The rest of paper is organized as follows. In section 2 the CSP is reviewed briefly and the relation between CSP and some other spatial filtering methods is described. We explain the data and the proposed method in section 3. Results are represented in section 4 . Concluding remarks is brought in section 5 .

\section{BACKGROUND}

Here we briefly explain about CSP in the context of EEG signal processing $[2,3]$. Let us assume the observation data as $\mathbf{X} \in \mathbb{R}^{N \times T}$, where $N$ is the number of channels (electrode leads) and $T$ is the number of time samples. Each row of $\mathbf{X}$ represents the zero mean signal of EEG channels. We consider two classes of data related to two states of brain as $C^{1}$ and $C^{2}$. Let us assume $T^{\ell}, \ell=1,2$ as the time intervals re- 
lated to each class as follows:

$$
\mathbf{X}^{\ell}=\mathbf{X}\left[T^{\ell}\right] \in C^{\ell}
$$

where,

$$
\mathbf{X}\left[T^{\ell}\right]= \begin{cases}\mathbf{X}[i] & i \in T^{\ell} \\ 0 & i \notin T^{\ell}\end{cases}
$$

The goal of CSP is to find a linear transform $\mathbf{W}$ to maximize the variance difference between $\mathbf{W}^{T} \mathbf{X}^{1} \in C^{1}$ and $\mathbf{W}^{T} \mathbf{X}^{2} \in C^{2}$. Equivalently, we project the data to maximize the power during $T^{1}$ and minimize the power during $T^{2}$ [5]. This can be formulated as the following optimization problem:

$$
\max _{\mathbf{W}} \frac{\mathbf{w}^{T} \widehat{\mathbf{R}}^{1} \mathbf{w}}{\mathbf{w}^{T} \widehat{\mathbf{R}}^{2} \mathbf{w}} \text { s.t. }\|\mathbf{w}\|=1
$$

where,

$$
\widehat{\mathbf{R}}^{\ell}=\frac{1}{M^{\ell}} \mathbf{X}^{\ell} \mathbf{X}^{\ell^{T}}, M^{\ell}=\operatorname{length}\left(T^{\ell}\right)
$$

Using Rayleigh-Ritz theorem solving (3) leads to generalized eigenvalue decomposition (GEVD) of $\left(\widehat{\mathbf{R}}^{1}, \widehat{\mathbf{R}}^{2}\right)$ :

$$
\widehat{\mathbf{R}}^{1} \mathbf{W}=\widehat{\mathbf{R}}^{2} \mathbf{W} \Lambda
$$

where $\Lambda$ is the diagonal matrix of eigenvalues. The eigenvalues are ranked in decreasing order.

Here we briefly explain about the link between CSP and other spatial filtering approaches.

1) It has been shown in [4] that CSP in a two-class problem can be approximated as the maximization of the mutual information between ICA components and class labels, where class labels $b^{\ell}(t)$ can be considered as:

$$
b^{\ell}[i]=\left\{\begin{array}{ll}
1 & i \in T^{\ell} \\
0 & i \notin T^{\ell}
\end{array} .\right.
$$

2) The relationship between CSP and ML was investigated in [3]. The following model

$$
\mathbf{X}^{\ell}=\mathbf{A} \mathbf{S}^{\ell}+\mathbf{E}^{\ell}
$$

was considered for each class $\ell$, where $\mathbf{A}^{\ell}, \mathbf{S}^{\ell}$ and $\mathbf{E}^{\ell}$ are the mixing matrix, sources, and additive noise, respectively. It has been shown that $\mathbf{W}$ in CSP is equal to $\widehat{\mathbf{A}}^{-T}$.

3) According to [6], in the BSS methods based on GEVD instead of conventional statistics like covariance matrices, any other a priori information can be used. Therefore, CSP using GEVD of $\left(\widehat{\mathbf{R}}^{1}, \widehat{\mathbf{R}}^{2}\right)$ can be considered as semi-blind source separation (SBSS) method. In fact, CSP is equivalent to source separation methods based on non-stationarity [7], considering data in only two time windows.

\section{MATERIALS AND METHODS}

\subsection{Data}

The iEEG recordings ${ }^{1}$ were obtained from a 31 year old male with left temporal lobe epilepsy. The standard clinical diagnosis using his scalp EEG showed an interictal spiking activity involved not only in the left temporal lobe, but also in the basal frontal and insular cortex. The patient underwent evaluation for resective surgery with the iEEG recordings. He is seizure free after left temporal lobectomy surgery.

Twelve semi-rigid multi-lead intracerebral electrodes with $0.8 \mathrm{~mm}$ diameter were bilaterally implanted in suspected seizure origins based on clinical considerations. The multilead electrodes (Dixi, Besançon, France) include 5, 10, 15 or 18 leads evenly spaced every $1.5 \mathrm{~mm}$. Each lead has 2 $\mathrm{mm}$ length. The iEEG were recorded with an audio-videoEEG monitoring system (Micromed, Treviso, Italy) with a maximum of 128 depth-EEG channels and sampled at 512 Hz. We focus on bipolar derivations since they may provide more correctly measure differences of potentials between two recording electrodes [8].

The $50 \mathrm{~Hz}$ is removed by a 5-order notch Butterworth filter with $3 \mathrm{~dB}$ lower, and upper band stop frequencies equal to $48 \mathrm{~Hz}$ and $52 \mathrm{~Hz}$, respectively.

\subsection{Method}

The method consists of two steps 1) IED and non-IED detection, and 2) application of CSP. In the following we explain each of these steps.

\subsubsection{IED and non-IED detection}

The IED and non-IED time intervals are labeled on the temporal iEEG signals. This stage has been done manually by the epileptologist. Typical IED and non-IED time intervals from iEEG recordings are depicted in Figure 1.

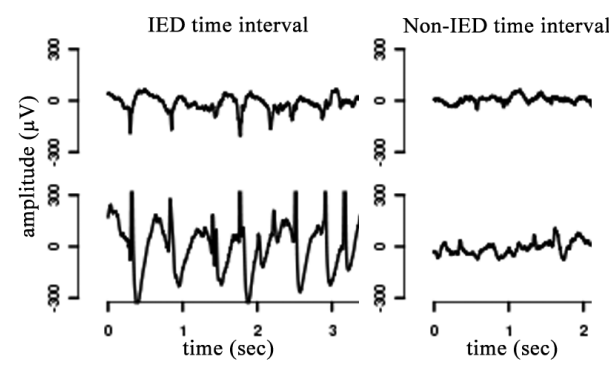

Fig. 1. Typical IED and non-IED time intervals.

We have $N$-dimensional observations $\mathbf{X}=\left[\begin{array}{lll}\mathbf{x}_{1} & \ldots & \mathbf{x}_{N}\end{array}\right]^{T}$, where $\mathbf{x}_{i}$, is the $i$ th row of matrix $\mathbf{X} \in \mathbb{R}^{N \times T}$. $\mathbf{x}_{i}$ contains $T$ samples of the signal recorded from the $i$ th channel, and

\footnotetext{
${ }^{1}$ EEG recordings are provided by P. Kahane, head of Neurology Department, CHUG, Grenoble, France.
} 
$N$ is the number of channels associated with bipolar iEEG electrode leads.

A 5-order Butterworth band-pass filter is applied on the rows of matrix $\mathbf{X}$. The lower and upper band stop frequencies of the filter are equal to $2 \mathrm{~Hz}$, and $64 \mathrm{~Hz}$, respectively. This frequency range is selected since the power of iEEG signals during IED time intervals has high contribution in this range. The filtered signals are segmented according to IED and nonIED labels providing $L^{1}+L^{2}$ number of matrices denoted as $\mathbf{X}_{m}^{\ell}$, where $m=1, \ldots, L^{\ell}$. In the following $\ell=1$ for IED and $\ell=2$ for non-IED state. Matrix $\mathbf{X}_{m}^{\ell}$ has $T_{m}^{\ell}$ columns, where $T_{m}^{\ell}$ is the length of $m$ th IED or non-IED time interval denoted as $\mathbf{x}_{i m}^{\ell} . i=1, \ldots, N$ is the channel index. The above procedure is depicted in the block diagram of figure 2 .

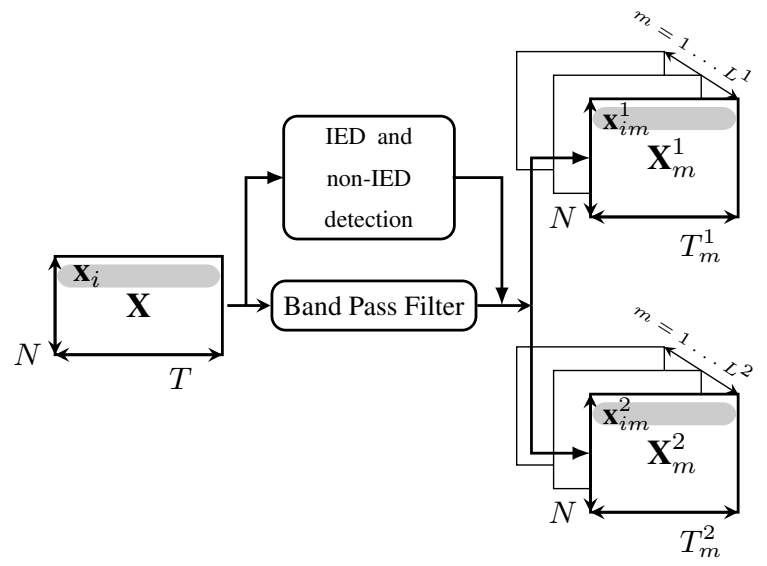

iEEG signals

IED and non-IED

segment matrices

Fig. 2. IED and non-IED segment matrices.

\subsubsection{Application of CSP}

The scheme depicted in figure 3 demonstrates the block diagram of applying CSP method. Up to now we segmented the filtered iEEG signals to $L^{1}$ IED and $L^{2}$ non-IED segment matrices denoted as $\mathbf{X}_{m}^{1}$, and $\mathbf{X}_{m}^{2}$, respectively. We calculate the correlation matrix for each of IED or non-IED matrices using (4) by substituting $\mathbf{X}^{\ell}$ with $\mathbf{X}_{m}^{\ell}$. These correlation matrices are denoted as $\mathbf{R}_{m}^{\ell} \in \mathbb{R}^{N \times N}, m=1, \ldots, L^{\ell}$. The average of $L^{1}$ IED correlation matrices and $L^{2}$ non-IED correlation matrices are calculated providing two matrices denoted as $\mathbf{R}^{1}$ and $\mathbf{R}^{2}$ for IED and non-IED, respectively. $\mathbf{R}^{1}$ and $\mathbf{R}^{2}$ are the input of GEVD. In GEVD, the linear transformation matrix $\mathbf{W}$ is obtained according to the decreasing order of the eigenvalues. Assuming the following linear model:

$$
\mathbf{X}=\mathbf{A S},
$$

the spatial patterns and temporal sources are calculated in terms of $\mathbf{W}$ as:

$$
\begin{gathered}
\mathbf{A}=\mathbf{W}^{-T} \\
\mathbf{S}=\mathbf{W}^{T} \mathbf{X} .
\end{gathered}
$$

We assume $\mathbf{A}=\left[\mathbf{a}_{1} \ldots \mathbf{a}_{N}\right], \mathbf{a}_{i}=\left[a_{i}[1] \ldots a_{i}[N]\right]^{T}$ and $\mathbf{S}=\left[\begin{array}{lll}\mathbf{s}_{1} & \ldots & \mathbf{s}_{N}\end{array}\right]^{T} . \mathbf{a}_{i}$ and $\mathbf{s}_{i}$ are the $i$ th spatial pattern and temporal source, respectively. $a_{i}[j]$ shows the contribution of the $i$ th source in the $j$ th electrode lead. The temporal sources, $\mathbf{s}_{i}$, and their corresponding spatial patterns, $\mathbf{a}_{i}$, are ranked according to the decreasing power of IED intervals. Assuming the number of sources mostly related to the IED class is known, we threshold the corresponding spatial patterns. The electrode leads whose spatial pattern values (absolute value of $\left.a_{i}[j]\right)$ are greater than a predefined threshold are considered as the estimated IED regions. Here we obtain the number of mostly related sources using a thresholding method which is explained in section 4 . However, the number of sources could be obtained from prior knowledge.

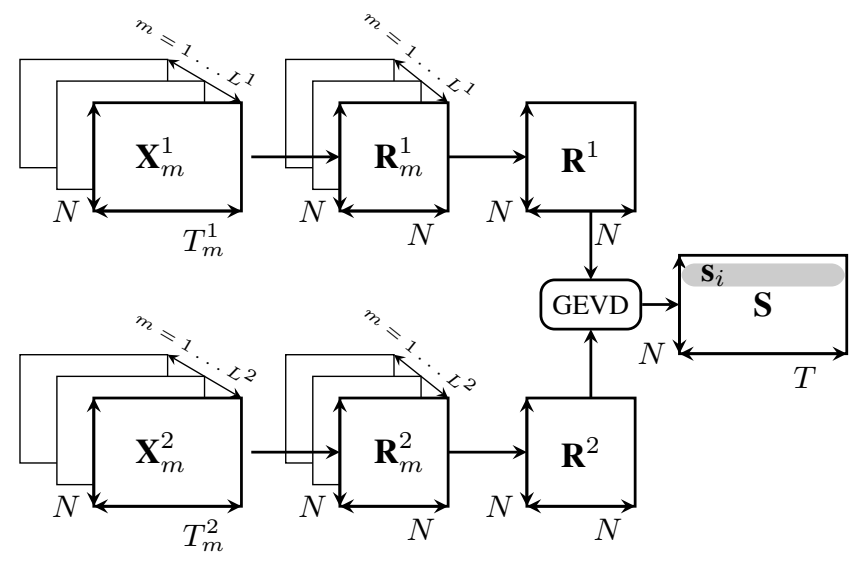

Fig. 3. Common Spatial Pattern (CSP)

\section{RESULTS}

Number of IED and non-IED time intervals are $L^{1}=L^{2}=$ 195. Number of iEEG channels, $N$, and the length of data processed, $T$, are equal to 111 and $129 \times 10^{4}$ samples (about 42 minutes), respectively. The sampling rate, $f s$, is equal to $512 \mathrm{~Hz}$.

The method is applied on the iEEG recordings of one patient. Following the interpretation of $a_{i}[j]$ given in section 3.2.2, $a_{i}[j]$ shows the contribution of the $i$ th source in the $j$ th electrode lead. In figure $4, \mathbf{s}_{1}, \mathbf{s}_{2}, \mathbf{a}_{1}$, and $\mathbf{a}_{2}$ are shown. According to parts (c)-(d) of figure 4 , it can be seen that the first and second sources have high contributions $\left(\left|a_{i}[j]\right|>10\right)$ in electrode leads $\{79,80\}$ and $\{47,67,68,69,79\}$, respectively. Electrode leads $\{47\},\{67,68,69\}$, and $\{79,80\}$ are located in parahippocampal gyrus $(p H c G)$, anterior hippocampus $(a n t H C)$, and posterior hippocampus (post $H C$ ), respectively. The electrode leads with high relation (i.e. $\left.\left|a_{i}[j]\right|\right)$ to the first two $^{2}$ sources are considered as the estimated IED regions. Therefore $p H c G$, ant $H C$ and post $H C$ regions are considered as the estimated IED regions. The

\footnotetext{
${ }^{2}$ The reason of selecting the first two sources is explained later in this Section.
} 
visually inspected SOZs of the patient by the epileptologist correspond to the same three regions.

For verifying that the sources are ranked according to their contribution to IED class, we compute the ratio of the power of sources in IED time intervals to the power of sources in non-IED time intervals [5]. The ratio, called power ratio, $P R$ of the $i$ th source can be defined as:

$$
P R_{i}=\frac{\sum_{b^{1}[k]=1}\left(\mathbf{s}_{i}[k]\right)^{2}}{\sum_{b^{2}[k]=1}\left(\mathbf{s}_{i}[k]\right)^{2}}
$$

where $b^{\ell}[k]$ is defined in (6). The percentage of normalized $P R_{i}$ values for the first five temporal sources are reported in table 4. The more relevant sources according to CSP ranking the greater $P R_{i}$ values. To select the number of relevant sources, a threshold equal to $10 \%$ is chosen. Therefore, to estimate the IED regions the first two sources are selected.

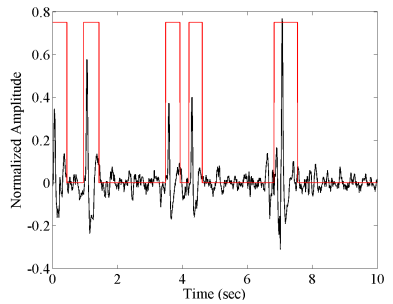

(a) 1 st source

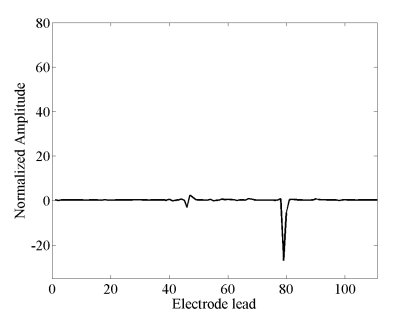

(c) Spatial pattern of the 1st source

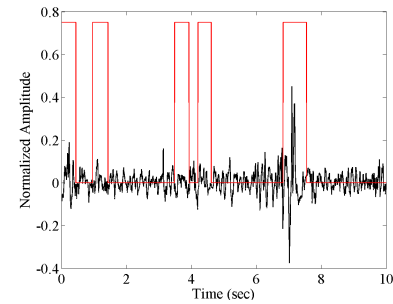

(b) 2nd source

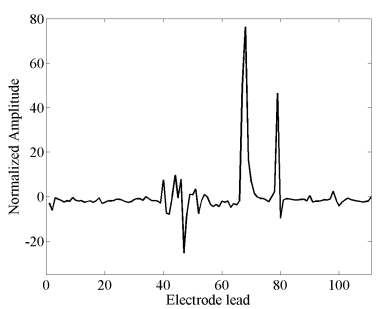

(d) Spatial pattern of the 2nd source
Fig. 4. Space-time sources. (a) First and (b) second temporal sources for 10 seconds. IED time intervals corresponding to temporal iEEG signals are determined on temporal sources with a step function. Spatial patterns corresponding to (c) first and (d) second temporal sources.

Table 1. Normalized power ratio $(P R)$ for the first five temporal sources.

\begin{tabular}{lccccc}
\hline Source index & 1 & 2 & 3 & 4 & 5 \\
\hline$P R$ & 67.4 & 13.5 & 8.9 & 6.2 & 4 \\
\hline
\end{tabular}

\section{CONCLUSION}

A method for identification of IED regions from iEEG recordings based on CSP is proposed. Basically, this method is well suited for separating the discriminative sources between two brain states: here, IED and non-IED. The two-class CSP method is fast and simple since its solution is based on GEVD which is an analytical mathematic problem. Here, the processing time for about 195 IED and non-IED time intervals and 111 channels is about 4 minutes (using a shared $3 \mathrm{GHz}, 4$ core Xeon 64 bits processor). Moreover, it is robust provided that covariance matrices are accurately estimated [3], that we have achieved due to the large number of IED and non-IED time intervals.

The proposed method is applied on the iEEG recordings of one seizure-free patient after resective surgery. The estimated IED regions were congruent with the visually inspected SOZ by the epileptologist for this patient. Although we need additional patients for further investigations, this result is encouraging. The proposed method has two drawbacks. First, the detection of IED and non-IED time intervals is done manually. Automatic detection of IED and non-IED time intervals can be considered as our first perspective. Secondly, the method is threshold-based. A second perspective would be to define rigorously the threshold. Finally it is also necessary to apply the method on a few patients.

\section{REFERENCES}

[1] F. Rosenow and H. Lüders, "Presurgical Evaluation Of Epilepsy," Brain, vol. 124, no. 9, pp. 1683-1700, 2001.

[2] K. Fukunaga and W. L. G. Koontz, "Application of the k-1 expansion to feature selection and ordering," IEEE Trans. on Computers, , no. 4, pp. 311-318, 1970.

[3] W. Wu, Z. Chen1, S. Gao, and E. N. Brown, "A Probabilistic Framework for Learning Robust Common Spatial Patterns," in Annual International Conference of the IEEE EMBS, 2009.

[4] M. Grosse-Wentrup and M. Buss, "Multiclass Common Spatial Patterns and Information Theoretic Feature Extraction," IEEE Trans. on BME, vol. 55, pp. 1991-2000, 2008.

[5] L.C. Parra, C.D. Spence, A.D. Gerson, and P. Sajda, "Recipes for the Linear Analysis of EEG," Neuroimage, vol. 28, pp. 326-341, 2005.

[6] R. Sameni, C. Jutten, and M.B. Shamsollahi, "Multichannel Electrocardiogram Decomposition Using Periodic Component Analysis," IEEE Trans. on BME, vol. 55, no. 8, pp. $1935-1940,2008$.

[7] J.F. Cardoso and D. Pham, "Separation of non-stationary sources: algorithms and performance," in Independent Component Analysis: Principles and Practice, Stephen J. Roberts and Ricahrd M. Everson, Eds., pp. 158-180. Cambridge University Press, mar 2001.

[8] P. L. Nunez and R. Srinivasan, Electric Fields of the Brain: The Neurophysics of EEG, Oxford University Press, Inc., 2006. 